\title{
Peptidoglycan Salvage Enables the Periodontal Pathogen Tannerella forsythia to Survive within the Oral Microbial Community
}

\author{
Isabel Hottmann ${ }^{a} \quad$ Marina Borisova ${ }^{a} \quad$ Christina Schäffer ${ }^{b} \quad$ Christoph Mayer $^{a}$ \\ a Interfaculty Institute of Microbiology and Infection Medicine, Organismic Interactions/Glycobiology, Eberhard Karls \\ Universität Tübingen, Tübingen, Germany; ${ }^{b}$ Department of NanoBiotechnology, NanoGlycobiology Unit, Universität \\ für Bodenkultur Wien, Vienna, Austria
}

\section{Keywords}

Oral biofilm - Periodontitis - Pathogen $\cdot N$-acetylmuramic acid $\cdot$ Peptidoglycan . Cell wall recycling

\begin{abstract}
Tannerella forsythia is an anaerobic, fusiform Gram-negative oral pathogen strongly associated with periodontitis, a multibacterial inflammatory disease that leads to the destruction of the teeth-supporting tissue, ultimately causing tooth loss. To survive in the oral habitat, $T$. forsythia depends on cohabiting bacteria for the provision of nutrients. For axenic growth under laboratory conditions, it specifically relies on the external supply of $\mathrm{N}$-acetylmuramic acid (MurNAc), which is an essential constituent of the peptidoglycan (PGN) of bacterial cell walls. T. forsythia comprises a typical Gramnegative PGN; however, as evidenced by genome sequence analysis, the organism lacks common enzymes required for the de novo synthesis of precursors of PGN, which rationalizes its MurNAc auxotrophy. Only recently insights were obtained into how T. forsythia gains access to MurNAc in its oral habitat, enabling synthesis of the own PGN cell wall. This re-
\end{abstract}

port summarizes T. forsythia's strategies to survive in the oral habitat by means of PGN salvage pathways, including recovery of exogenous MurNAc and PGN-derived fragments but also polymeric PGN, which are all derived from cohabiting bacteria either via cell wall turnover or decay of cells. Salvage of polymeric PGN presumably requires the removal of peptides from PGN by an unknown amidase, concomitantly with the translocation of the polymer across the outer membrane. Two recently identified exo-lytic $\mathrm{N}$-acetylmuramidases (Tf_NamZ1 and Tf_NamZ2) specifically cleave the peptide-free, exogenous (nutrition source) PGN in the periplasm and release the MurNAc and disaccharide substrates for the transporters Tf_MurT and Tf_AmpG, respectively, whereas the peptide-containing, endogenous (the self-cell wall) PGN stays unattached. This review also outlines how $T$. forsythia synthesises the PGN precursors UDP-MurNAc and UDP-Nacetylglucosamine (UDP-GlcNAc), involving homologs of the Pseudomonas sp. recycling enzymes AmgK/MurU and a monofunctional uridylyl transferase (named Tf_GlmU*), respectively.
(C) 2021 The Author(s)

Published by S. Karger AG, Basel karger@karger.com www.karger.com/mip

Karger $\stackrel{\text { ' }}{5}$
(C) 2021 The Author(s)

Published by S. Karger AG, Basel

This is an Open Access article licensed under the Creative Common Attribution-NonCommercial-4.0 International License (CC BY-NC) (http://www.karger.com/Services/OpenAccessLicense), applicable to the online version of the article only. Usage and distribution for commercial purposes requires written permission.
Correspondence to:

Christoph Mayer, christoph.mayer@uni-tuebingen.de 


\section{Introduction}

Tannerella forsythia, originally designated Bacteroides forsythius, is an anaerobic Gram-negative bacterium taxonomically grouped to the Porphyromonadaceae within the Bacteroidetes phylum [Tanner et al., 1986; Maiden et al., 2003]. It was first described by Anne Tanner at the Boston Forsyth Institute as a "slow growing fusiform $\mathrm{Bac}$ teroides" and was isolated from apical plaque samples from patients suffering from advanced periodontitis [Tanner et al., 1979; Tanner et al., 1986]. Remarkably, the bacterium could not be obtained in pure culture, but it grew in the presence of a helper bacterium, such as Fusobacterium nucleatum, even when the two bacteria were separated by a semipermeable membrane [Tanner et al., 1986; Dzink et al., 1987]. This indicated that survival of T. forsythia depends on an essential growth factor that is released from the helper bacterium. This mysterious compound was subsequently identified by Chris Wyss at the University of Zürich as $N$-acetylmuramic acid (MurNAc), which supported axenic growth of $T$. forsythia [Wyss, 1989]. Together with $N$-acetylglucosamine (GlcNAc), MurNAc is an essential carbohydrate constituent of the peptidoglycan (PGN) of the bacterial cell wall. The glycan backbone of PGN is built from these two carbohydrates, which are connected via $\beta-1,4$ glycosidic bonds in an alternate arrangement and multiple of these glycan chains are cross-linked via short peptides linked to MurNAc [Weidel and Pelzer, 1964; Walter and Mayer,

Fig. 1. Overview of the PGN/MurNAc salvage and biosynthetic pathways of $T$. forsythia crucial for its survival within polymicrobial biofilms. T. forsythia lacks homologs of GlmS, GlmM, a bifunctional GlmU, MurA, and MurB (light gray), which are generally essential enzymes in bacteria, generating UDP-GlcNAc and UDP-MurNAc required for PGN biosynthesis. The later steps of PGN biosynthesis via MurCDEF, MurI, Alr, Ddl, MraY, and MurG are present in T. forsythia (brown). Hence, T. forsythia depends on the salvage of external MurNAc-sources. Exogenous MurNAc (for symbols refer to legend) and GlcNAc-MurNAc/GlcNAc-anhMurNAc disaccharides are internalized via the inner membrane (IM) transporters Tf_MurT (MurT) and Tf_AmpG (AmpG), respectively. Translocation across the outer membrane (OM) is supposedly facilitated via SusC-like TonB-dependent uptake machineries that work in complex with SusD-like glycan-binding lipoproteins. Uptake of both, (anhydro-)muropeptides and longer PGN chains, presumably occurs by concomitant removal of the stem peptides by a so far unknown PGN amidase, thereby releasing peptide-free disaccharides and PGN-glycans (poly-GlcNAc-MurNAc chains) into the periplasm. The removal of the peptide side chains from the PGN upon entry in the cell likely allows T. forsythia to distinguish between exogenous (nutrition source) and endogenous (the
2019]. Thereby a flexible mesh-like structure is generated, the PGN sacculus, that allows the bacterial cell to withstand intracellular osmotic pressure (turgor), thus protecting from lysis [Vollmer and Seligman, 2010]. T. forsythia possesses a typical Gram-negative PGN [Mayer et al., 2019b]. However, genome analysis of T. forsythia revealed that the bacterium lacks orthologs of murA/murB and $\mathrm{glmS} / \mathrm{glmM} / \mathrm{glm} U$ (shown in Fig. 1; proteins missing in T. forsythia but commonly present in bacteria are depicted in gray). These genes encode generally essential enzymes for the de novo biosynthesis of the PGN precursors UDP-MurNAc and UDP-GlcNAc [Chen et al., 2005; Friedrich et al., 2015]. Specifically, due to the lack of murA/murB, T. forsythia is unable to synthesize UDPMurNAc from UDP-GlcNAc, rationalizing the MurNAc auxotrophy of $T$. forsythia. Moreover, due to the lack of $\mathrm{glmS} / \mathrm{glmM} / \mathrm{glmU}, T$. forsythia is also unable to synthesize UDP-GlcNAc from fructose 6-phosphate (fructose 6P) (Fig. 1).

Since T. forsythia apparently fails to de novo synthesize UDP-MurNAc and UDP-GlcNAc, it depends on the salvage of MurNAc and, presumably, also of GlcNAc from the environment to synthesize its cell wall. It is conceivable to assume that the polymicrobial flora in the oral cavity, harboring more than 700 bacterial species [Lamont et al., 2018], provides to $T$. forsythia distinct options to access various sources of MurNAc and GlcNAc. Indeed, the ability to establish itself in a polymicrobial biofilm consortium, commonly known as dental plaque, is supposed

self-cell wall) PGN within the periplasm. Peptide-free polyGlcNAc-MurNAc chains are readily degraded in the periplasm by the recently identified paralogous exo- $N$-acetylmuramidases $\mathrm{Tf}$ NamZ1 and Tf_NamZ2, that act on peptide-free PGN-glycans releasing GlcNAc-MurNAc disaccharides and MurNAc, respectively. Subsequently, these cleavage products are translocated across the IM by the transporters Tf_AmpG and Tf_MurT, respectively. PGN-derived disaccharides may be cleaved by an $\mathrm{N}$-acetylglucosaminidase (Tf_NagZ) (cf. Table 1) within the cytoplasm. MurNAc can either be shuttled into the PGN synthesis pathway via the Tf_ AmgK/Tf_MurU enzymes of T. forsythia (blue) thereby generating UDP-MurNAc, or enters the PGN catabolic pathway via Tf MurK/Tf_MurQ (green), leading to formation of GlcNAc 6P, which is further catabolized by Tf_NagA and Tf_NagB. Tf_AmgK may phosphorylate GlcNAc, besides MurNAc, as shown for the Pseudomonas homolog [Gisin et al., 2013]. In this work, we report on the discovery of a monofunctional GlcN 1P uridylyl transferase (GlmU*) that converts GlcNAc $1 P$ to UDP-GlcNAc. Thus, T. forsythia is able to bypass the missing PGN de novo biosynthetic enzymes MurA and MurB (gray) by using salvage pathways for exogenous PGN and fragments thereof (red and blue).

(For figure see next page.) 
to be a prerequisite for the survival of $T$. forsythia [Bloch et al., 2019]. Formation of multispecies dental plaque biofilms follows a clear spatiotemporal scheme and its progression plays a crucial role in periodontitis. Periodontitis is a multifactorial inflammatory disease, characterized by the irreversible destruction of the teeth-supporting tissue and leading to tooth loss if untreated [Holt and Ebersole, 2005]. A distinction is drawn between the different colonizers and stages of maturation of dental plaque biofilms, according to which the so-called "early colonizers," which are mainly Gram-positive streptococci, precondition the oral surfaces for the subsequent adherence and growth of so called "bridge bacteria" [Socransky et al., 1998; Kolenbrander, 2000]. During biofilm maturation, these "bridge bacteria," including Fusobacterium nucleatum, create a suitable environment for the so-called "late colonizers" [Diaz et al., 2000; 2002]. Among these are the periodontal pathogens T. forsythia, the related Bacteroidetes species Porphyromonas gingivalis, and the spirochete Treponema denticola, which together build up the so-called "red complex" [Paster et al., 2006]. The late colonizing red complex consortium is associated with severe forms of

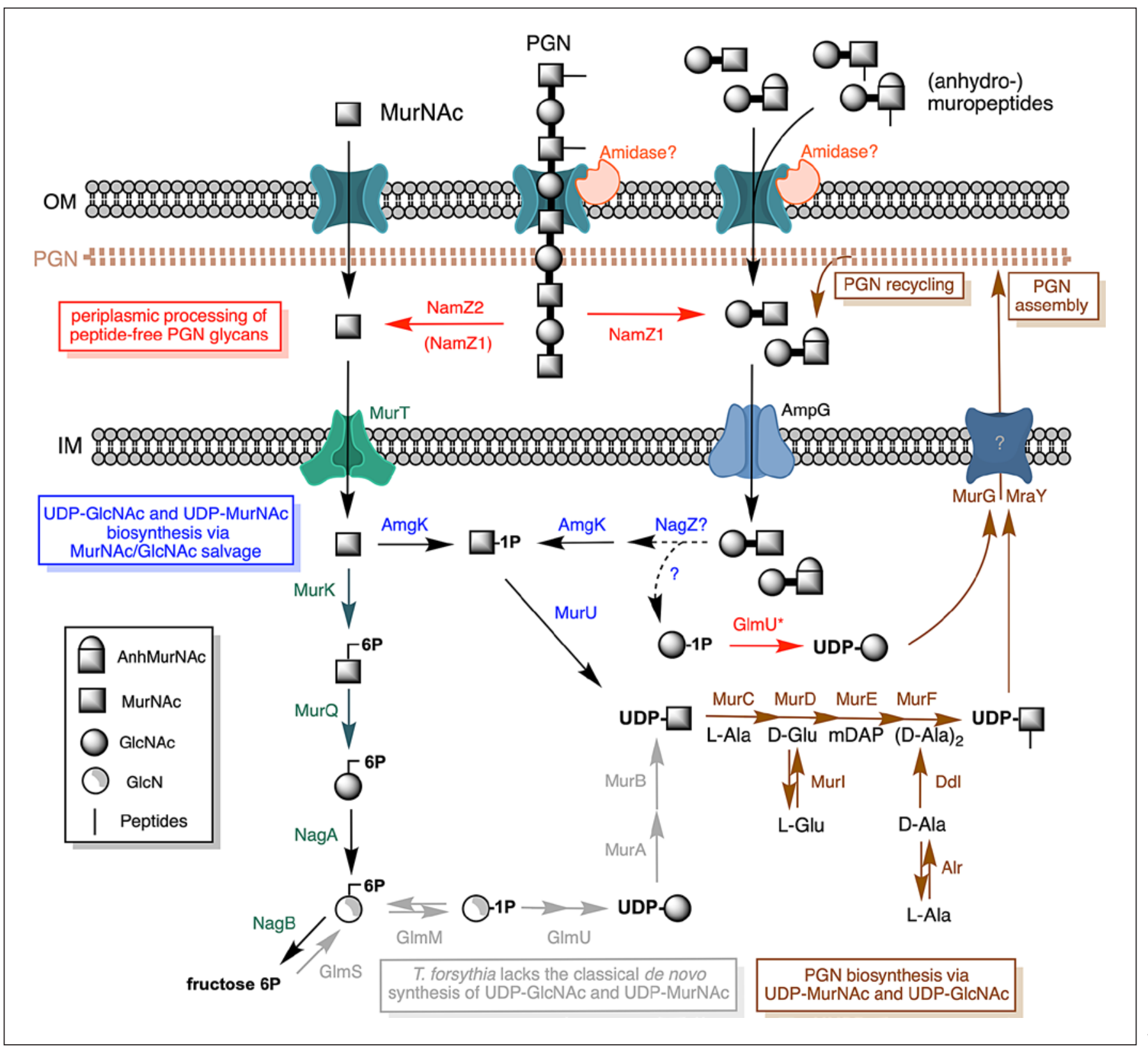

Tannerella forsythia Peptidoglycan

Microb Physiol 2021;31:123-134

DOI: $10.1159 / 000516751$ 
periodontitis [Holt and Ebersole, 2005]. Given the fastidious growth requirements of T. forsythia in a laboratory monoculture, the question arises, which natural sources the bacterium can exploit to ensure its survival in the oral cavity. In this review, we describe the most recent advances in the understanding of the salvage pathways used by $T$. forsythia within oral microbial communities to acquire the essential growth factor MurNAc and how this is metabolized to allow PGN biosynthesis.

\section{PGN Type and Cell Envelope Structure of T. forsythia}

As a Gram-negative bacterium, T. forsythia contains a thin, moderately crosslinked PGN layer located within the periplasm, embedded between the inner membrane and the lipopolysaccharide (LPS)-containing outer membrane. A recent compositional analysis of the PGN of $T$. forsythia revealed the typical carbohydrate building blocks GlcNAc and MurNAc as well as the 1,6-anhydroform of MurNAc (anhMurNAc), constituting the terminal ends of the PGN chains [Mayer et al., 2019b]. T. forsythia PGN was assigned to the classical A1 $\gamma$-type PGN of Gram-negative bacteria with meso-diaminopimelic acid $(m \mathrm{DAP})$ involved in direct crosslinks [Schleifer and Kandler, 1972; Mayer et al., 2019b]. The LPS of T. forsythia is of the rough type and contains the membrane anchored lipid A, inner and outer core - but lacks a distal O-specific antigen [Raetz and Whitfield, 2002; Posch et al., 2013]. A characteristic of the cell envelope of $T$. forsythia is the additional coverage of the outer membrane by a two-dimensional crystalline surface (S)-layer, consisting of self-assembling glycoproteins (Tf_TfsA and Tf TfsB) [Lee et al., 2006; Posch et al., 2011b; Sekot et al., 2012]. A glycosylated S-layer is rare among Gram-negative bacteria and characterizes different $T$. forsythia strains, containing either a pseudaminic acid or legionaminic acid derivative at the non-reducing terminal end of a complex S-layer oligosaccharide. These sialic acidlike nonulosonic acids (9-carbon backbone 2-keto acid sugars) were shown to influence pathogenicity of the bacterium [Posch et al., 2011a; Friedrich et al., 2017; Bloch et al., 2019] and their synthesis was shown to depend on either UDP-GlcNAc or GDP-GlcNAc, respectively, as precursors [Schoenhofen et al., 2006; Schoenhofen et al., 2009; Friedrich et al., 2017]. Since T. forsythia constantly builds up its complex cell envelope during growth, the organism relies on the supply of a suitable amino sugar precursors for an efficient synthesis of its complex cell wall glycoconjugates, including LPS, S-layer glycopro- teins, and importantly, it relies on the supply of UDPGlcNAc and UDP-MurNAc for efficient synthesis of its PGN.

\section{PGN Biosynthesis in T. forsythia in Comparison to Other Bacteria and Characterization of GImU*}

Given the presence of multiple amino sugars in its different cell envelope structures, $T$. forsythia surprisingly lacks generally essential proteins of the amino sugar biosynthetic pathway. The bacterium lacks a glucosamine 6-phosphate synthase (glutamine-fructose 6P amidotransferase; GlmS), which converts the fructose 6-phosphate intermediate of glycolysis to glucosamine 6-phosphate (GlcN 6P) [Badet et al., 1987]. This enzyme is the initial amino sugar biosynthetic enzyme of most bacteria, indicating that $T$. forsythia is unable to de novo synthesize GlcN 6P and, hence, amino sugars derived from it. This enzyme is also the main regulator of amino sugar metabolism in other bacteria. To adjust the cellular level of GlcN $6 \mathrm{P}$ to the growth requirements, $\mathrm{glmS}$ expression is controlled via a GlmS-riboswitch in the Gram-positive bacterium Bacillus subtilis and via small regulatory RNAs and the sensor protein RapZ in the Gram-negative bacterium Escherichia coli [Klein and Ferre-D'Amare, 2006; Kalamorz et al., 2007]. Since T. forsythia lacks GlmS, the regulation of amino sugar metabolism has to be regulated in a different way in this organism. The presence of the subsequent enzyme of the general amino sugar anabolic pathway, which isomerizes GlcN 6P to glucosamine $\alpha-1$ phosphate (GlcN 1P) [Mengin-Lecreulx and van Heijenoort, 1996], is uncertain in T. forsythia. A glucosaminephosphate isomerase (Tf_GlmM; BFO_1882; Tanf_ 04345) annotated in the genome of T. forsythia reveals higher similarity to phosphogluco-/phosphomannomutases (e.g., 30\% amino acid identity with AlgC of Pseudomonas putida) than to GlmM-like phosphoglucosamine mutases. In most bacteria, GlcN 1P is further converted to UDP-GlcNAc by a bifunctional $\mathrm{N}$-acetyltransferase/ uridylyl transferase (GlmU), which comprises an N-terminal GlcN 1P acetyltransferase domain and a C-terminal GlcNAc 1P uridylyl transferase domain [Mengin-Lecreulx and van Heijenoort, 1994; Gehring et al., 1996]. T. forsythia lacks a bifunctional UDP-GlcNAc synthase [Hottmann et al., 2018; Ruscitto and Sharma, 2018]. We found, however, that the organism possesses a monofunctional GlcNAc 1P uridylyl transferase (designated Tf_GlmU*; BFO_1878; Tanf_04325), which is a homolog of a monofunctional Cc_GlmU* $(43 \%$ overall amino acid 


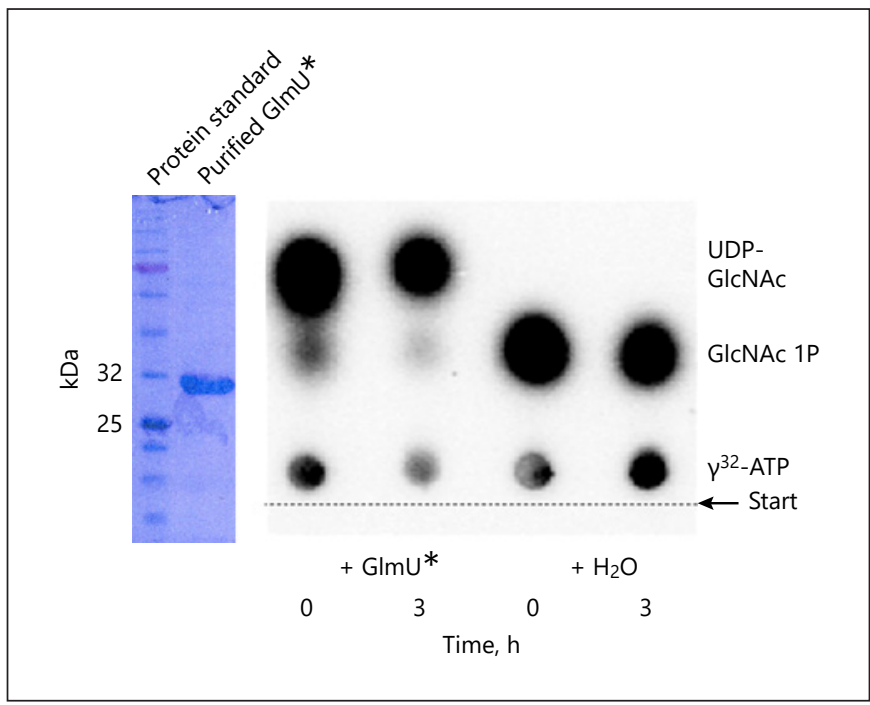

Fig. 2. Purity and activity of recombinant Tf_GlmU* analyzed by SDS-PAGE and by UDP-GlcNAc formation using a radioactive enzyme assay. Left: Coomassie stained 12\% SDS-PAGE gel; lane 1, protein standard mixture with molecular masses as indicated; lane 2, purified, recombinant GlmU*. Right: Radioactive assay of recombinant GlmU*. [ $\left.{ }^{32} \mathrm{P}\right]-\mathrm{GlcNAc} \alpha-1 \mathrm{P}$ (GlcNAc $\left.1 \mathrm{P}\right)$ was generated from GlcNAc and $\left[{ }^{32} \mathrm{P}\right]$-ATP using an anomeric MurNAc/ GlcNAc kinase (AmgK from P. putida KT2440) [Gisin et al., 2013; Renner-Schneck et al., 2015]. Furthermore, $\left[{ }^{32} \mathrm{P}\right]-$ GlcNAc $a-1 \mathrm{P}$ (GlcNAc 1P) was incubated with $5 \mu \mathrm{g}$ of Tf_GlmU* and $50 \mathrm{~mm}$ UTP (control with water instead of enzyme) and reactions were spotted on a TLC plate immediately (0) and after $3 \mathrm{~h}(3)$ of incubation at $37^{\circ} \mathrm{C}$.

sequence identity to Ccan_15070) that has been identified in the oral pathogen Capnocytophaga canimorsus [Renzi et al., 2015]. C. canimorsus is auxotrophic for GlcNAc (not MurNAc), but is able to salvage this amino sugar from salivary mucins present in the oral cavity. As an adaption to the availability of GlcNAc in the oral bacterium's settling, the dog mouth, C. canimorsus apparently has lost its capability to de novo synthesize UDPGlcNAc, hence, its genome has lost the genes encoding GlmM and the bifunctional GlmU [Renzi et al., 2015].

To determine the function of Tf_GlmU*, the 309-amino acid recombinant enzyme containing a C-terminal His $_{6}$-tag was expressed in E. coli BL21 (DE3) cells. The protein was purified using nickel-affinity chromatography, followed by size exclusion chromatography (using HiLoad 16/60 Superdex 200 column, GE Healthcare) to near homogeneity (shown in Fig. 2). With a radioactive uridylyl transferase assay as described earlier [Gisin et al., 2013; Renner-Schneck et al., 2015], the Tf_GlmU*-reac- tion was analyzed using $\left[{ }^{32} \mathrm{P}\right]-\mathrm{GlcNAc} \alpha$-1-phosphate (GlcNAc 1P) as a substrate (shown in Fig. 2; also see material and methods). After the addition of GlmU* and UTP, we observed the direct conversion of GlcNAc 1P to UDP-GlcNAc, which was not detectable in the negative control. For an unequivocal product determination, a reaction mixture with non-labeled GlcNAc $1 \mathrm{P}$ was also analyzed by electrospray-ionization time-of-flight (ESI-TOF) mass spectrometry as described earlier [Gisin et al., 2013] (shown in Fig. 3). The substrate GlcNAc 1P appeared with a mass of $(\mathrm{M}-\mathrm{H})^{-}=300.063 \mathrm{~m} / z$ in negative ion mode, which is in accordance with the theoretical mass of GlcNAc $1 \mathrm{P}\left((\mathrm{M}-\mathrm{H})^{-}=300.049 \mathrm{~m} / z\right)$ (Fig. 3; in blue). After incubation with Tf_GlmU*, the substrate was partly converted into a product with $(\mathrm{M}-\mathrm{H})^{-}=606.100 \mathrm{~m} / z$, which is in agreement with the expected mass of UDP-GlcNAc ((M$\left.\mathrm{H})^{-}=606.074 \mathrm{~m} / \mathrm{z}\right)$ (Fig. 3b; in orange)).

The biosynthesis of PGN is a conserved process within bacteria that can be divided into three major steps occurring in different cell compartments. In the first step, the soluble precursor molecules UDP-MurNAc and UDPMurNAc-pentapeptide are synthesized from UDPGlcNAc in the cytoplasm (shown in Fig. 1). In the second step, the undecaprenyl-phosphate-anchored PGN precursors lipid I and lipid II are synthesized at the cytoplasmic membrane, which, in the last step, are assembled to polymeric, crosslinked PGN in the periplasm. UDP-MurNAc, the first dedicated precursor of PGN, is synthesized from UDP-GlcNAc via the enzymes MurA and MurB, representing the known route for MurNAc synthesis in nature. In the first reaction, MurA catalyzes the transfer of an enolpyruvate from phosphoenolpyruvate to the 3 '-hydroxyl group of UDP-GlcNAc. The thereby generated UDP-GlcNAc-enolpyruvate is subsequently reduced by MurB using one reduction equivalent of NADPH to generate UDP-MurNAc [Benson et al., 1993]. In T. forsythia, these first committed steps in the biosynthesis of PGN are not possible due to the lack of the initiating enzymes MurA/MurB. All the following reactions leading to UDP-MurNAc-pentapeptide (MurC-F, Ddl, MurI, Alr) and the further reactions leading to lipid II (MraY, MurG), are present in T. forsythia (shown in Fig. 1). These reactions are responsible for the sequential ATP-dependent ligation of single amino acids. MurC (BFO_0400; Tanf_11285) transfers L-alanine to UDP-MurNAc, and MurD (BFO_0403; Tanf_11300) and MurE (BFO_0405; Tanf_11310) further add D-glutamic acid (generated by the L-/D-glutamate racemase MurI; BFO_1448; Tanf_09790) and mDAP, yielding UDP-MurNAc-tripeptide. Finally, MurF (BFO_1349; Tanf_06315) is re- 


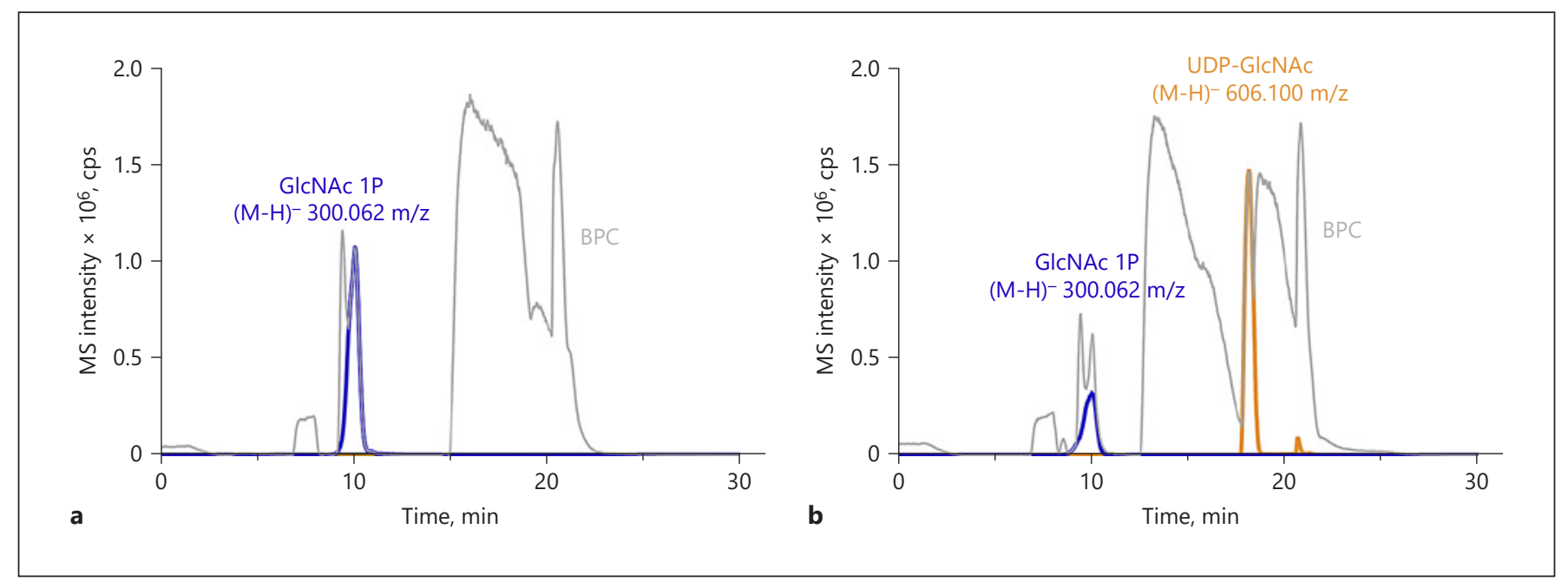

Fig. 3. Analysis of uridylyl transferase activity of recombinant $\mathrm{Tf}$ GlmU* by LC-MS. The reaction mixture containing GlcNAc 1P in Tris- $\mathrm{HCl}$ buffer ( $\mathrm{pH}$ 7.6) was analyzed prior (a) and after addition of UTP and purified recombinant Tf_GlmU* of T. forsythia (b). Shown are the base peak chromatograms (BPCs; grey) and extract-

quired for the addition of the dipeptide D-Ala-D-Ala, which in generated by ligation of two D-alanine molecules by the ligase Ddl (BFO_3290; Tanf_02600), yielding UDP-MurNAc-pentapeptide [Smith, 2006]. In the second step, the membrane-anchored precursors lipid I and lipid II are synthesized by MraY (BFO_0404; Tanf_11305) and MurG (BFO_0401; Tanf_11290), respectively (shown in Fig. 1). The prenyl sugar transferase MraY firstly transfers phospho-MurNAc-pentapeptide to the lipid carrier undecaprenyl phosphate, generating undecaprenyl pyrophosphoryl-MurNAc-pentapeptide, named lipid I [AlDabbagh et al., 2008]. Subsequently, lipid II is generated by the action of the glycosyltransferase MurG that transfers a GlcNAc moiety from UDP-GlcNAc onto lipid I [Bouhss et al., 2004]. In the final third step, lipid II is translocated to the outer leaflet of the cytoplasmic membrane, which is mediated by MurJ-like flippases belonging to the multidrug/oligosaccharidyl-lipid/polysaccharide (MOP) exporter superfamily, allowing the assembly of PGN by PGN-synthases [Typas et al., 2012; Sham et al., 2014; Cho et al., 2016]. Surprisingly, T. forsythia lacks an obvious MurJ homolog, and it is thus unclear how lipid II is translocated in this organism. Importantly, however, an undecaprenyl-phosphate carrier system is present, as $T$. forsythia contains both an undecaprenyl diphosphate synthase (UppS; BFO_1444; Tanf_09810) and an undecaprenyl diphosphatase (UppP/BacA, BFO_1764; Tanf_03865). Additionally, homologs of FtsW ed ion chromatograms (EICs) for GlcNAc 1P (blue) and UDPGlcNAc (orange). The measured mass-to-charge ratios of the compounds, indicated in the figure, are in agreement with the theoretical $\mathrm{m} / z$ values of $(\mathrm{M}-\mathrm{H})^{-}=300.049 \mathrm{~m} / z$ for GlcNAc $1 \mathrm{P}$ and of $(\mathrm{M}-\mathrm{H})^{-}=606.074 \mathrm{~m} / z$ for UDP-GlcNAc.

(BFO_0400; Tanf_11295) and RodA (BFO_3355; Tanf_08125) can be found on the T. forsythia genome, as well as Rod-complex and divisome components and penicillin-binding protein PGN synthases.

\section{MurNAc Uptake and Metabolism in T. forsythia: Evidence for an AmgK-MurU Salvage Route}

Since $T$. forsythia cannot de novo synthesize UDPMurNAc due to the lack of murA and murB orthologs (Fig. 1), it relies on the salvage of exogenous MurNAc or alternative sources of MurNAc. As mentioned above, $T$. forsythia's dependency on external MurNAc was first reported by Wyss in 1989, who observed that exogeneous MurNAc is a crucial growth factor and required for the maintenance of a rod-shaped (fusiform) cell morphology [Wyss, 1989]. We confirmed the MurNAc-dependency of T. forsythia and the transition from healthy rod-shaped to fusiform cells upon MurNAc starvation [Hottmann et al., 2018]. This observation clearly showed that $T$. forsythia must utilize an uptake system for MurNAc (and other MurNAc-sources) and a biosynthesis pathway leading to UDP-MurNAc that substitutes for MurA and MurB.

The ability to recover components of their own cell wall is a common feature of bacteria. During growth of bacteria, the cell wall undergoes a permanent turnover, and the released breakdown products are recovered in a 
process known as PGN recycling [Mayer et al., 2019a]. In Gram-negative bacteria, PGN recycling involves the cleavage of the PGN sacculus by lytic transglycosylases generating anhydromuropeptides (GlcNAc-anhMurNAc-peptides) which are efficiently recovered via the AmpG transporter [Mayer et al., 2019a]. Some bacteria can grow on PGN components provided as nutrients. For instance, in E. coli, the uptake of MurNAc is mediated by a phosphotransferase system (PTS) transporter that imports and simultaneously phosphorylates MuNAc, yielding MurNAc 6P [Dahl et al., 2004]. The typical PTS transports and phosphorylates sugars via the concerted action of the cytoplasmatic enzymes EI (enzyme I) and HPr (histidine protein), and the EII complex, which is composed of the sugar-specific domains EIIA and EIIB, and the transmembrane domain EIIC) [Siebold et al., 2001]. MurNAc 6P is the substrate of MurQ, a key enzyme of cell wall recycling, i.e., degradation of anhydromuropeptides, and MurNAc metabolism, which converts MurNAc 6P to GlcNAc 6P [Jaeger et al., 2005; Borisova et al., 2016]. Genome analysis of $T$. forsythia revealed the presence of an ortholog of murQ but not of murP [Ruscitto et al., 2016]. In view of the genetic context, $T f \_m u r Q$ is part of a threegene cluster encoding a putative sodium solute transporter (Tf_MurT; BFO_0041; Tanf_08375), a putative sugar kinase (Tf_MurK; BFO_0042; Tanf_08380), and the putative MurNAc 6P lactyl ether hydrolase MurQ (Tf MurQ; BFO_0044; Tanf_08385). The Tf_MurT transporter was characterized as a PTS-independent MurNAc transporter, the first known MurNAc uptake system of this kind, and Tf_MurK and Tf_MurQ were characterized as a specific MurNAc kinase and a bona fide MurNAc 6P lacyl ether hydrolase, respectively [Ruscitto et al., 2016; Hottmann et al. 2018]. The clustered organization of the murTKQ genes seems quite common among organisms from the Bacteroidetes phylum [Ruscitto et al., 2016; Ruscitto and Sharma, 2018].

For the metabolization of sugars their phosphorylation is mandatory. As MurNAc is not phosphorylated in T. forsythia by the transporter MurT, the concomitance of a sugar kinase (Tf_MurK) also encoded within the murTKQ cluster is consequential [Ruscitto et al., 2016; Hottmann et al., 2018]. Biochemical investigations of kinase activity of MurK revealed stringent specificity for MurNAc phosphorylation at the position C6, thereby generating MurNAc 6P, the substrate for the etherase MurQ and catabolism [Hottmann et al., 2018]. The phosphorylation position of MurNAc represents a metabolic branching point in $T$. forsythia. Intriguingly, the deletion of $T f \_$murK $\left(\Delta T f \_m u r K\right)$ results in the accumulation of

Tannerella forsythia Peptidoglycan

Salvage two metabolites, MurNAc and the PGN precursor molecule UDP-MurNAc-pentapeptide [Hottmann et al., 2018]. The accumulation of the latter provided that MurNAc can also be channeled into the anabolic PGN synthesis route as evidenced by a growth advantage of a $T$. forsythia $\Delta$ murK mutant in MurNAc-depleted growth medium [Hottmann et al., 2018].

Pseudomonas spp. possess an alternative route for the synthesis of MurNAc that bypasses the de novo biosynthesis of UDP-MurNAc and recovers MurNAc directly as UDP-MurNAc [Gisin et al., 2013]. This pathway includes, first, the phosphatase MupP, which dephosphorylates MurNAc 6P forming MurNAc [Borisova et al., 2017]. Next, the anomeric kinase AmgK phosphorylates the amino sugars GlcNAc and MurNAc forming GlcNAc $\alpha-1 P$ an MurNAc $\alpha-1 P$, respectively. Subsequently, the a-1-phosphorylated sugars can be used by GlmU and the uridylyl transferase MurU for the generation of UDPGlcNAc and UDP-MurNAc, respectively. Biochemical characterization of MurU of $P$. putida revealed stringent specificity for the uridylation of MurNAc $\alpha-1 P$ and not GlcNAc $\alpha$-1P [Renner-Schneck et al., 2015]. Similar functions might be provided by the orthologs Tf_amgK and Tf_murU found in T. forsythia (unpublished data). Most likely, the Tf_AmgK/Tf_MurU pathway is the principal route for PGN synthesis in T. forsythia and replaces the missing MurA/MurB pathway. The deficit of genes is a phenomenon reported from other bacteria that thrive in biofilms. For so far unknown reason, this might provide an increased fitness [Koskiniemi et al., 2012].

\section{T. forsythia Salvages PGN Turnover Products from Cohabiting Bacteria}

T. forsythia grows on MurNAc, yet anhydromuropeptides (GlcNAc-AnhMurNAc-peptides) and muropeptides (GlcNAc-MurNAc-peptides) are more commonly found in the natural environment [Fujimoto and Fukase, 2011]. Anhydromuropeptides constitute the general PGN turnover products of bacteria, while muropeptides represent PGN degradation products resulting from the activity of lysozyme-like muramidases [Mayer et al., 2019a]. Thus, it is reasonable to assume that T. forsythia salvages anhydromuropeptides and muropeptides which both likely occur in oral biofilms. Anhydromuropeptides are generated in large amounts during cell wall turnover, whereby the PGN layer is steadily cleaved by autolytic enzymes (autolysins), namely lytic transglycosylases and endopeptidases to enable bacterial growth [Höltje, 1995; 
Table 1. Occurrence of selected recycling/turnover enzymes in T. forsythia and cohabiting bacteria

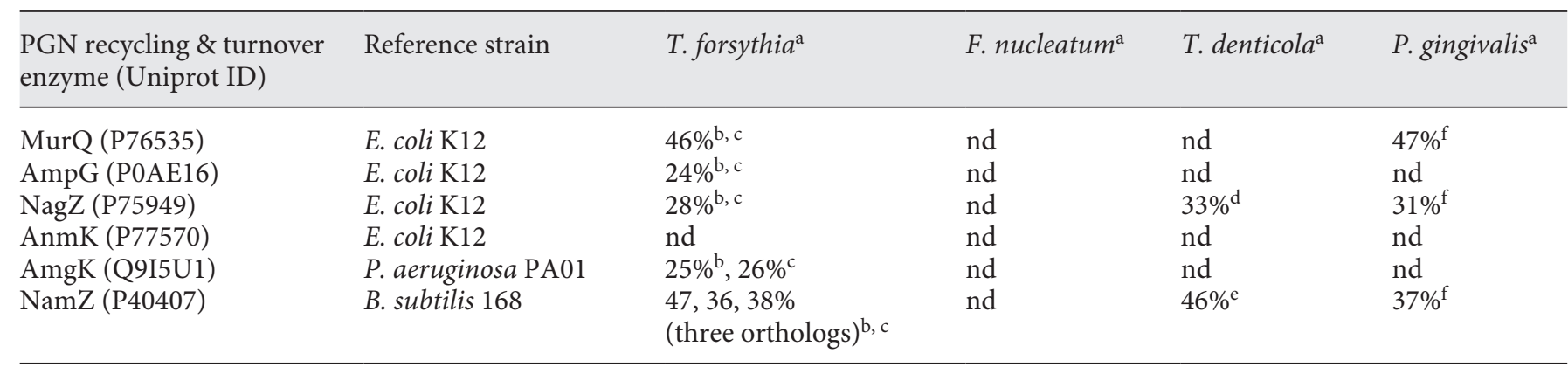

${ }^{a}$ BlastP analysis was performed using the non-redundant protein sequences database for searching of orthologs in the genome of $T$. forsythia 92A2 (taxid: 203275) and ATCC 43037 (accession number JUET00000000 [Friedrich et al., 2015]), F. nucleatum (taxid: 851), T. denticola (taxid: 158) and P. gingivalis (taxid: 837). Identity percentages relative to the indicated query protein are shown for orthologs from ${ }^{\mathrm{b}}$ T. forsythia strain 9 A2A, ${ }^{\mathrm{c}}$ T. forsythia stain ATCC 43037, ${ }^{\mathrm{d}}$ T. denticola strain ATCC $35405,{ }^{\mathrm{e}}$ T. denticola strain SP3, ${ }^{\mathrm{f}} P$. gingivalis strain W83. nd, orthologs not detected.

van Heijenoort, 2011; Mayer et al., 2019a]. In E. coli, per generation, about $50 \%$ of the PGN of the cell wall is cleaved by autolysins releasing anhydromuropeptides into the periplasm, from where they are efficiently internalized (recycled) by the inner membrane permease AmpG [Park and Uehara, 2008; Mayer et al., 2019a]. While in T. forsythia, a AmpG transporter homolog (BFO_0039; Tanf_08365) was identified, several cohabitating bacteria of $T$. forsythia (shown in Table 1), such as $F$. nucleatum, $T$. denticola, and $P$. gingivalis, lack orthologs of the E. coli AmpG permease. This indicates that $T$. forsythia might benefit from PGN turnover products released by these bacteria within the oral biofilm. Although Tf_AmpG shows rather low (24\%) sequence identity to $E$. coli AmpG [Ruscitto et al., 2017], its function as an anhydromuropeptide permease was initially proposed on the basis the ability of Tf_ampG to complement an E. coli ampDampG mutant using a reporter assay [Ruscitto et al., 2017]. Subsequently, we showed that T. forsythia grows on anhydromuropeptides derived from E. coli PGN cleaved with a lytic transglycosylase, while a $T$. forsythia $\triangle a m p G$ deletion mutant cannot [Mayer et al., 2020]. Intriguingly, we also revealed Tf_AmpG to be responsible for growth in the presence of muropeptides derived from muramidase-cleaved PGN of either F. nucleatum or $E$. coli [Mayer et al., 2020]. Surprisingly, growth on muropeptides was clearly favoured over that on anhydromuropeptides, as evidenced by a final OD about twice as high with muropeptides as compared to identical amounts of anhydromuropeptides [Mayer et al., 2020]. Further, we could show that peptide-free disaccharides, i.e. GlcNAcanhMurNAc and GlcNAc-MurNAc generated by pre- treatment of the (anhydro-)muropeptides with a recombinant $E$. coli amidase (AmiD), yielded identical growth rates compared with the peptide-containing (anhydro-) muropeptides. Thus, apparently, an endogenous amidase in T. forsythia is able to remove the peptide portions from the (anhydro)-muropeptides, yielding disaccharides. Intriguingly, the T. forsythia $\triangle a m p G$ mutant, in contrast to the T. forsythia wild-type, did not grow in medium supplemented with the peptide-free disaccharides GlcNAcMurNAc and GlcNAc-anhMurNAc. This distinguishes the T. forsythia AmpG-ortholog from E. coli AmpG, as the specificity of the latter is restricted to anhydromuropeptides and GlcNAc-anhMurNAc but does not accept GlcNAc-MurNAc [Cheng and Park, 2002].

\section{PGN Serves as MurNAc-Source for T. forsythia}

An exciting recent finding was that $T$. forsythia could also use intact PGN as a growth factor [Mayer et al., 2020]. PGN from the cohabiting bacteria $P$. gingivalis and $F$. $n u$ cleatum, from E. coli, as well as from T. forsythia, served as a growth factor for T. forsythia, replacing MurNAc or (anhydro-)muropeptides. In contrast, the $T$. forsythia $\Delta a m p G$ mutant was severely impaired in growth when cultured with intact PGN instead of MurNAc, indicating that transport via AmpG is critical to sustain growth on PGN [Mayer et al., 2020]. This observation implies that T. forsythia is able to degrade PGN mainly to GlcNAcMurNAc or GlcNAc-anhMurNAc disaccharides rather than to MurNAc. In our group, we recently identified and characterized a novel exo- $\mathrm{N}$-acetylmuramidase in $\mathrm{B}$. sub- 
tilis named NamZ [Müller et al., 2021]. This enzyme releases MurNAc from glycoside substrates carrying a MurNAc at the non-reducing end, such as the chromogenic reporter substrate para-nitrophenyl-MurNAc (pNPMurNAc), the natural PGN-derived disaccharide MurNAc-GlcNAc as well as peptide-free PGN chains terminating with a MurNAc residue. NamZ was found to constitute the founding member of a novel family of exo-acting PGN $\beta$ - $N$-acetylmuramidases [Müller et al., 2021]. In the light of MurNAc-auxotrophy of T. forsythia, we reasoned that the presence of enzymes exhibiting exo$\beta$ - $N$-acetylmuramidase activity could be beneficial. Intriguingly, the genome of $T$. forsythia encodes three orthologs of B. subtilis NamZ (Tf_NamZ1: BFO_0040; Tanf_08370; Tf_NamZ2: BFO_2140; Tanf_00660, and Tf_NamZ3: BFO_2092; Tanf_00855; see Table 1). Furthermore, NamZ orthologs were also found in P. gingivalis as well as in T. denticola (Table 1), consistent with the finding that the NamZ family glycosidases (CAZy family GH171; www.cazy.org/GH171) are prominently found within the members of the Fibrobacteres-Chlorobi-Bacteroidetes FCB superphylum [Müller et al., 2021]. The T. forsythia NamZ2 was shown to have a similar function to NamZ of B. subtilis. This enzyme cleaves peptide-free PGN glycans and MurNAc-glycosides with a MurNAc at the reducing end, and generates MurNAc, which can be transported into the cell via MurT (shown in Fig. 1). The other NamZ ortholog, Tf_NamZ1, was identified as a GlcNAc-MurNAc disaccharide-releasing exolytic $\beta-N$ acetylmuramidase. This enzyme also releases MurNAc by hydrolysis of MurNAc-glycosides, albeit less efficiently than NamZ2; its main function is the cleavage of peptidefree PGN yielding GlcNAc-MurNAc disaccharides (as well as GlcNAc-anhMurNAc disaccharides, which commonly resides at the reducing ends of the glycans). The nam $Z 1$ gene is located directly adjacent to $\operatorname{amp} G$ on the $T$. forsythia genome, which encodes the transporter of GlcNAc-MurNAc and GlcNAc-anhMurNAc [Mayer et al., 2020]. Intriguingly, the subsequent gene downstream of $a m p G$, lyt $B$, displays significant similarity to SpoIIDdomain containing lytic transglycosylases [Morlot et al., 2010]. Moreover, T. forsythia possesses at least three further putative lytic transglycosylases belonging to glycosyl hydrolase family 23 (www.cazy.org). These enzyme may be involved in trimming peptide-free, exogeneous PGN or be involved in PGN recycling in T. forsythia. In summary, the exo- $N$-acetylmuramidases Tf_NamZ1 and Tf_ NamZ2 presumably degrade peptide-free PGN fragments in the periplasm to yield GlcNAc-MurNAc (besides GlcNAc-anhMurNAc) and MurNAc, which are the sub-

Tannerella forsythia Peptidoglycan Salvage strates of the T. forsythia AmpG and MurT transporters, respectively. Based on our findings, we suggest that PGN is internalized by $T$. forsythia through the outer membrane, possibly via a SusCD-like uptake complex (shown in Fig. 1). Sus complexes are common uptake complexes within the polysaccharide utilization loci (PUL) of Bacteroidetes bacteria, consisting of TonB-dependent outer membrane transporter (SusC) and cell surface glycanbinding proteins (SusD) [Grondin et al., 2017; Lapebie et al., 2019]. An amidase, as mentioned earlier, is required to remove the peptides from PGN upon internalization. Peptide-free exogeneous PGN is then processed by NamZ1 and NamZ2 to yield GlcNAc-MurNAc/GlcNAcanhMurNAc and MurNAc (shown in Fig. 1) and subsequentyl these fragments are taken up via Tf_AmpG and Tf_MurT and are further processed intracellularly by shuttling to the UDP-MurNAc biosynthesis pathway, via Tf_AmgK and Tf_MurU, or through the catabolic pathway via Tf_MurK and Tf_MurQ (shown in Fig. 1).

\section{Conclusion}

The MurNAc auxotrophic oral pathogen T. forsythia compensates for its fastidious growth requirements in the oral cavity by salvaging of MurNAc, and MurNAccontaining PGN fragments such as GlcNAc-MurNAc and GlcNAc-anhydroMurNAc disaccharides as well as (anhydro-)muropeptides, which are common cell wall turnover products, presumably released into the environment by cohabiting bacteria. Intriguingly, we revealed that many of the cohabiting bacteria lack an ortholog of AmpG, and thus cannot recover the GlcNAc-MurNAc and GlcNAc-anhydroMurNAc disaccharides released from their PGN cell wall during turnover. Sometimes they even lack further important PGN recycling enzymes (MurQ, AnmK, AmgK; see Table 1). Thus, these bacteria likely provide $T$. forsythia with essential PGN turnover fragments. Moreover, T. forsythia is able to salvage intact PGN, which is processed by two members of a newly identified family of exo- $\beta-N$-acetylmuramidases (Tf NamZ1 and Tf_NamZ2) within the periplasm. Since these enzymes require peptide-free PGN as substrates, we suggest that the initial removal of the peptide stems from incoming PGN by an unknown amidase is critical to degrade exogeneous PGN within the periplasm. This might be a strategy of T. forsythia to distinguish between exogenous PGN (nutritional source) and endogenous PGN (i.e., its own cell wall), because the exo- $\beta-N$ acetylmuramidases specifically cleave peptide-free exo- 
genous PGN and leave the structural PGN unattached. Follow-up studies will identify the proposed amidase and clarify whether exogenous PGN is transported through the outer membrane via SusCD-like complexes. These complexes are clustered within so-called PULs and frequently used by Bacteroidetes to forage polysaccharides. However, a PGN-specific PUL has so far not been identified in the T. forsythia genome. Moreover, the roles of AmgK and MurU in PGN biosynthesis remains to be clarified. The understanding of cell wall and amino sugar metabolism in T. forsythia and the elucidation of the different MurNAc-containing sources and routes of uptake and metabolism used by this bacterium to survive within the oral microbial community will help to understand its physiological demands and set the basis to develop new approaches for the treatment of periodontitis.

\section{Materials and Methods/Experimental Procedures}

Plasmid Construction, Expression, and Purification of GlmU*

To determine the function of Tf_GlmU*, the encoding gene was amplified from genomic DNA of T. forsythia ATCC 43037 using the primer pair GlmU*fw_ TACGAACATATGAAACCAACATTATTTGTGCTGGCGGC/GlmU*rev_ GCGCTCGAGACCGAACAGCTGAGCGGGATATTC (introduced restriction sites are shown in bold) and cloned in vector pET29b for the expression as a C-terminally $\mathrm{His}_{6}$-tagged-fusion protein. The PCR product and vector pET29 were digested with NdeI and XhoI (NEB, Frankfurt, Germany) and ligated using T4 DNA ligase (Thermo Fisher Scientific, Waltham, MA, USA). The resulting plasmid (pET29_GlmU*) was transformed into E. coli BL21(DE3) cells for overproduction.

\section{Radioactive Uridylyl Transferase Assay}

The Tf_GlmU* reaction was analyzed with a radioactive uridylyl transferase assay using $\left[{ }^{32} \mathrm{P}\right]-$ GlcNAc $\alpha$-1-phosphate as a substrate, which was prepared from GlcNAc and $\gamma$ - $\left[{ }^{32} \mathrm{P}\right]$-ATP using AmgK from $P$. putida as described earlier [Gisin et al., 2013; Renner-Schneck et al., 2015]. For the radioactive uridylyl transferase assay, $5 \mu \mathrm{L}$ of the AmgK reaction mixture were incubated with $50 \mathrm{~mm}$ UTP and $1 \mu \mathrm{g}$ of Tf_GlmU* in $100 \mathrm{~mm}$ Tris-HCl buffer $(\mathrm{pH}$ 7.6) and spotted immediately and after $3 \mathrm{~h}$ of incubation at $37^{\circ} \mathrm{C}$ on a TLC plate (silica gel $60 \mathrm{~F}_{254}$; Merck, Darmstadt, Germany). After separation in h $n$-butyl alcohol:methanol:25\% (wt/vol) ammonium hydroxide:water (5:4:2:1), the TLC plate was analyzed using a Typhoon Trio Biomolecular Imager (GE Healthcare).

\section{LC-MS Uridylyl Transferase Assay}

A non-radioactive, LC-MS uridylyl transferase assay was performed as described above but using unlabeled GlcNAc $\alpha-1 P$ as the substrate, which was prepared according to a published protocol [Gisin et al., 2013]. $25 \mathrm{mM}$ of GlcNAc $\alpha-1 \mathrm{P}$ was used in a $100-\mu \mathrm{L}$ reaction mixture containing $100 \mathrm{mM}$ Tris- $\mathrm{HCl}(\mathrm{pH} \mathrm{7.6),} 50 \mathrm{mM}$ UTP, 1 U baker's yeast inorganic pyrophosphatase and $5 \mu \mathrm{g}$ of Tf_GlmU*. The reaction was incubated for $3 \mathrm{~h}$ at $37^{\circ} \mathrm{C}$. The reac- tion mixture was analyzed before and after the addition of GlmU* and $50 \mathrm{mM}$ UTP, using HPLC connected to an electrospray-ionization time-of-flight (ESI-TOF) mass spectrometer (Micro-TOF II; Bruker) [Gisin et al., 2013].

\section{Acknowledgement}

We thank Valentin Friedrich for very helpful discussions and Libera lo Presti for linguistic editing.

\section{Statement of Ethics}

Ethics approval was not required, as the work includes only an in vitro characterization of a carbohydrate metabolic enzyme.

\section{Conflict of Interest Statement}

The authors have no conflicts of interest to declare.

\section{Funding Sources}

This work was supported by the Deutsche Forschungsgemeinschaft (DFG; German Research Foundation, projects GRK1708/ B2 MA2436/7-1 and SFB766/A15; as well as the Germany' Excellence Strategy -EXC2124 - 390838134) and the Austrian Science Fund FWF (project I 2875-B22).

\section{Author Contributions}

I.H. performed all experiments. I.H., M.B., C.S. and C.M. conceived and wrote the manuscript.

References

Al-Dabbagh B, Henry X, El Ghachi M, Auger G, Blanot $D$, Parquet $C$, et al. Active site mapping of MraY, a member of the polyprenyl-phosphate N-acetylhexosamine 1-phosphate transferase superfamily, catalyzing the first membrane step of peptidoglycan biosynthesis. Biochemistry. 2008 Aug 26;47(34):891928.

Badet B, Vermoote P, Haumont PY, Lederer F, LeGoffic F. Glucosamine synthetase from Escherichia coli: purification, properties, and glutamine-utilizing site location. Biochemistry. 1987 Apr 7;26(7):1940-8.

Benson TE, Marquardt JL, Marquardt AC, Etzkorn FA, Walsh CT. Overexpression, purification, and mechanistic study of UDP-Nacetylenolpyruvylglucosamine reductase. Biochemistry. 1993 Mar 2;32(8):2024-30. 
Bloch S, Tomek MB, Friedrich V, Messner P, Schäffer C. Nonulosonic acids contribute to the pathogenicity of the oral bacterium Tannerella forsythia. Interface Focus. 2019 Apr 6; 9(2):20180064.

Borisova M, Gaupp R, Duckworth A, Schneider A, Dalügge D, Mühleck M, et al. Peptidoglycan recycling in Gram-positive bacteria is crucial for survival in stationary phase. mBio. 2016 Oct 11;7(5):e00923-16.

Borisova M, Gisin J, Mayer C. The N-acetylmuramic acid 6-phosphate phosphatase MupP completes the Pseudomonas peptidoglycan recycling pathway leading to intrinsic fosfomycin resistance. mBio. 2017 Mar 28;8(2).

Bouhss A, Crouvoisier M, Blanot D, Mengin-Lecreulx D. Purification and characterization of the bacterial MraY translocase catalyzing the first membrane step of peptidoglycan biosynthesis. J Biol Chem. 2004 Jul 16;279(29): 29974-80.

Chen T, Abbey K, Deng WJ, Cheng MC. The bioinformatics resource for oral pathogens. Nucleic Acids Res. 2005 Jul 1;33(Web Server issue):W734-40.

Cheng Q, Park JT. Substrate specificity of the AmpG permease required for recycling of cell wall anhydro-muropeptides. J Bacteriol. 2002 Dec;184(23):6434-6.

Cho H, Wivagg CN, Kapoor M, Barry Z, Rohs PDA, Suh H, et al. Bacterial cell wall biogenesis is mediated by SEDS and PBP polymerase families functioning semi-autonomously. Nat Microbiol. 2016 Sep 19;1:16172.

Dahl U, Jaeger T, Nguyen BT, Sattler JM, Mayer C. Identification of a phosphotransferase system of Escherichia coli required for growth on $\mathrm{N}$-acetylmuramic acid. J Bacteriol. $2004 \mathrm{Apr}$; 186(8):2385-92.

Diaz PI, Zilm PS, Rogers AH. The response to oxidative stress of Fusobacterium nucleatum grown in continuous culture. FEMS Microbiol Lett. 2000 Jun 1;187(1):31-4.

Diaz PI, Zilm PS, Rogers AH. Fusobacterium nucleatum supports the growth of Porphyromonas gingivalis in oxygenated and carbon-dioxide-depleted environments. Microbiology (Reading). 2002 Feb;148(Pt 2):467-72.

Dzink JL, Smith CM, Socransky SS. Development of a broth medium for Bacteroides forsythus. J Clin Microbiol. 1987 May;25(5):925.

Friedrich V, Janesch B, Windwarder M, Maresch D, Braun ML, Megson ZA, et al. Tannerella forsythia strains display different cell-surface nonulosonic acids: biosynthetic pathway characterization and first insight into biological implications. Glycobiology. 2017 Apr; 27(4):342-57.

Friedrich V, Pabinger S, Chen T, Messner P, Dewhirst FE, Schäffer C. Draft genome sequence of Tannerella forsythia type strain ATCC 43037. Genome Announc. 2015 Jun 11;3(3).

Fujimoto Y, Fukase K. Structures, synthesis, and human Nod1 stimulation of immunostimulatory bacterial peptidoglycan fragments in the environment. J Nat Prod. 2011 Mar 25;74(3): $518-25$.
Gehring AM, Lees WJ, Mindiola DJ, Walsh CT, Brown ED. Acetyltransfer precedes uridylyltransfer in the formation of UDP-N-acetylglucosamine in separable active sites of the bifunctional GlmU protein of Escherichia coli. Biochemistry. 1996 Jan 16;35(2):579-85.

Gisin J, Schneider A, Nägele B, Borisova M, Mayer C. A cell wall recycling shortcut that bypasses peptidoglycan de novo biosynthesis. Nat Chem Biol. 2013 Aug;9(8):491-3.

Grondin JM, Tamura K, Déjean G, Abbott DW, Brumer H. Polysaccharide Utilization Loci: Fueling Microbial Communities. J Bacteriol. 2017 Aug 1;199(15).

Holt SC, Ebersole JL. Porphyromonas gingivalis, Treponema denticola, and Tannerella forsythia: the "red complex", a prototype polybacterial pathogenic consortium in periodontitis. Periodontol 2000. 2005 Jun;38:72-122.

Höltje JV. From growth to autolysis: the murein hydrolases in Escherichia coli. Arch Microbiol. 1995 Oct;164(4):243-54.

Hottmann I, Mayer VMT, Tomek MB, Friedrich V, Calvert MB, Titz A, et al. N-Acetylmuramic acid (MurNAc) auxotrophy of the oral pathogen Tannerella forsythia: characterization of a MurNAc kinase and analysis of Its role in cell wall metabolism. Front Microbiol. 2018;9:19.

Jaeger T, Arsic M, Mayer C. Scission of the lactyl ether bond of $\mathrm{N}$-acetylmuramic acid by Escherichia coli "etherase". J Biol Chem. 2005 Aug 26;280(34):30100-6.

Kalamorz F, Reichenbach B, März W, Rak B, Görke B. Feedback control of glucosamine6-phosphate synthase GlmS expression depends on the small RNA GlmZ and involves the novel protein YhbJ in Escherichia coli. Mol Microbiol. 2007 Sep;65(6):1518-33.

Klein DJ, Ferré-D'Amaré AR. Structural basis of glmS ribozyme activation by glucosamine6-phosphate. Science. 2006 Sep 22;313(5794): 1752-6.

Kolenbrander PE. Oral microbial communities: biofilms, interactions, and genetic systems. Annu Rev Microbiol. 2000;54:413-37.

Koskiniemi S, Sun S, Berg OG, Andersson DI. Selection-driven gene loss in bacteria. PLoS Genet. 2012 Jun;8(6):e1002787.

Lamont RJ, Koo H, Hajishengallis G. The oral microbiota: dynamic communities and host interactions. Nat Rev Microbiol. 2018 Dec; 16(12):745-59.

Lapebie P, Lombard V, Drula E, Terrapon N, Henrissat B. Bacteroidetes use thousands of enzyme combinations to break down glycans. Nat Commun. 2019 May 3;10(1):2043.

Lee SW, Sabet M, Um HS, Yang J, Kim HC, Zhu $\mathrm{W}$. Identification and characterization of the genes encoding a unique surface (S-) layer of Tannerella forsythia. Gene. 2006 Apr 12; 371(1):102-11.

Maiden MF, Cohee P, Tanner AC. Proposal to conserve the adjectival form of the specific epithet in the reclassification of Bacteroides forsythus Tanner et al. 1986 to the genus Tannerella Sakamoto et al. 2002 as Tannerella forsythia corrig., gen. nov., comb. nov. request for an opinion. Int J Syst Evol Microbiol. 2003 Nov; 53(Pt 6):2111-2.

Mayer C, Kluj RM, Mühleck M, Walter A, Unsleber S, Hottmann I, et al. Bacteria's different ways to recycle their own cell wall. Int J Med Microbiol. 2019a Nov;309(7):151326.

Mayer VMT, Hottmann I, Figl R, Altmann F, Mayer C, Schäffer C. Peptidoglycan-type analysis of the $\mathrm{N}$-acetylmuramic acid auxotrophic oral pathogen Tannerella forsythia and reclassification of the peptidoglycan-type of Porphyromonas gingivalis. BMC Microbiol. 2019b Sep 2;19(1):200.

Mayer VMT, Tomek MB, Figl R, Borisova M, Hottmann I, Blaukopf M, et al. Utilization of different MurNAc sources by the oral pathogen Tannerella forsythia and role of the inner membrane transporter AmpG. BMC Microbiol. 2020 Nov 17;20(1):352.

Mengin-Lecreulx D, van Heijenoort J. Copurification of glucosamine-1-phosphate acetyltransferase and $\mathrm{N}$-acetylglucosamine1-phosphate uridyltransferase activities of Escherichia coli: characterization of the glmU gene product as a bifunctional enzyme catalyzing two subsequent steps in the pathway for UDP-N-acetylglucosamine synthesis. Bacteriol. 1994 Sep;176(18):5788-95.

Mengin-Lecreulx D, van Heijenoort J. Characterization of the essential gene glmM encoding phosphoglucosamine mutase in Escherichia coli. J Biol Chem. 1996;271(1):32-9.

Morlot C, Uehara T, Marquis KA, Bernhardt TG, Rudner DZ. A highly coordinated cell wall degradation machine governs spore morphogenesis in Bacillus subtilis. Genes Dev. 2010 Feb 15;24(4):411-22.

Müller M, Calvert M, Hottmann I, Kluj RM, Teufel T, Balbuchta K, et al. The exo- $\beta-\mathrm{N}$ acetylmuramidase NamZ from Bacillus subtilis is the founding member of a family of exolytic peptidoglycan hexosaminidases. J Biol Chem. 2021 Mar 5; 100519.

Park JT, Uehara T. How bacteria consume their own exoskeletons (turnover and recycling of cell wall peptidoglycan). Microbiol Mol Biol Rev. 2008 Jun;72(2):211-contents.

Paster BJ, Olsen I, Aas JA, Dewhirst FE. The breadth of bacterial diversity in the human periodontal pocket and other oral sites. Periodontol 2000. 2000;42:80-7.2006

Posch G, Andrukhov O, Vinogradov E, Lindner B, Messner P, Holst O, et al. Structure and immunogenicity of the rough-type lipopolysaccharide from the periodontal pathogen Tannerella forsythia. Clin Vaccine Immunol. 2013 Jun;20(6):945-53.

Posch G, Pabst M, Brecker L, Altmann F, Messner P, Schäffer C. Characterization and scope of S-layer protein O-glycosylation in Tannerella forsythia. J Biol Chem. 2011a Nov 4;286(44): 38714-24.

Posch G, Pabst M, Brecker L, Altmann F, Messner P, Schäffer C. Characterization and scope of S-layer protein O-glycosylation in Tannerella forsythia. J Biol Chem. 2011b Nov 4;286(44): 38714-24. 
Raetz CR, Whitfield C. Lipopolysaccharide endotoxins. Annu Rev Biochem. 2002;71:635-700. doi: $\quad$ 10.1146/annurev.biochem.71.110601. 135414. Epub 2001 Nov 9.

Renner-Schneck M, Hinderberger I, Gisin J, Exner T, Mayer C, Stehle T. Crystal structure of the $\mathrm{N}$-acetylmuramic acid $\alpha$-1-phosphate (MurNAc- $\alpha 1-\mathrm{P}$ ) uridylyltransferase MurU, a minimal sugar nucleotidyltransferase and potential drug target enzyme in Gram-negative pathogens. J Biol Chem. 2015 Apr 24;290(17): 10804-13.

Renzi F, Manfredi P, Dol M, Fu J, Vincent S, Cornelis GR. Glycan-foraging systems reveal the adaptation of Capnocytophaga canimorsus to the dog mouth. MBio. 2015 Mar 3;6(2): e02507.

Ruscitto A, Honma K, Veeramachineni VM, Nishikawa K, Stafford GP, Sharma A. Regulation and Molecular Basis of Environmental Muropeptide Uptake and Utilization in Fastidious Oral Anaerobe Tannerella forsythia. Front Microbiol. 2017;8:648.

Ruscitto A, Hottmann I, Stafford GP, Schäffer C Mayer C, Sharma A. Identification of a novel $\mathrm{N}$-acetylmuramic acid transporter in Tannerella forsythia. J Bacteriol. 2016 Nov 15; 198(22):3119-25.

Ruscitto A, Sharma A. Peptidoglycan synthesis in Tannerella forsythia: scavenging is the modus operandi. Mol Oral Microbiol. 2018 Apr; 33(2):125-32.
Schleifer KH, Kandler O. Peptidoglycan types of bacterial cell walls and their taxonomic implications. Bacteriol Rev. 1972;36(4):407-77.

Schoenhofen IC, McNally DJ, Brisson JR, Logan SM. Elucidation of the CMP-pseudaminic acid pathway in Helicobacter pylori: synthesis from UDP-N-acetylglucosamine by a single enzymatic reaction. Glycobiology. $2006 \mathrm{Sep}$; 16(9):8C-14C.

Schoenhofen IC, Vinogradov E, Whitfield DM, Brisson JR, Logan SM. The CMP-legionaminic acid pathway in Campylobacter: biosynthesis involving novel GDP-linked precursors. Glycobiology. 2009 Jul;19(7):715-25.

Sekot G, Posch G, Oh YJ, Zayni S, Mayer HF, Pum $\mathrm{D}$, et al. Analysis of the cell surface layer ultrastructure of the oral pathogen Tannerella forsythia. Arch Microbiol. 2012 Jun;194(6):52539.

Sham LT, Butler EK, Lebar MD, Kahne D, Bernhardt TG, Ruiz N. Bacterial cell wall. MurJ is the flippase of lipid-linked precursors for peptidoglycan biogenesis. Science. 2014 Jul 11; 345(6193):220-2

Siebold C, Flükiger K, Beutler R, Erni B. Carbohydrate transporters of the bacterial phosphoenolpyruvate:sugar phosphotransferase system (PTS). FEBS Lett. 2001 Aug 31;504(3): 104-11.

Smith CA. Structure, function and dynamics in the mur family of bacterial cell wall ligases. J Mol Biol. 2006 Sep 29;362(4):640-55.

Socransky SS, Haffajee AD, Cugini MA, Smith C, Kent RL Jr. Microbial complexes in subgingival plaque. J Clin Periodontol. 1998 Feb;25(2): $134-44$
Tanner AC, Haffer C, Bratthall GT, Visconti RA, Socransky SS. A study of the bacteria associated with advancing periodontitis in man. J Clin Periodontol. 1979 Oct;6(5):278-307.

Tanner ACR, Listgarten MA, Ebersole JL, Strezempko MN. Bacteroides forsythus sp. nov, a slow-growing, fusiform Bacteroides sp. from the human oral cavity. Int J Syst Bacteriol. 1986 Apr;36(2):213-21.

Typas A, Banzhaf M, Gross CA, Vollmer W. From the regulation of peptidoglycan synthesis to bacterial growth and morphology. Nat Rev Microbiol. 2012 Dec 28;10(2):123-36.

van Heijenoort J. Peptidoglycan hydrolases of Escherichia coli. Microbiol Mol Biol Rev. 2011 Dec;75(4):636-63.

Vollmer W, Seligman SJ. Architecture of peptidoglycan: more data and more models. Trends Microbiol. 2010 Feb;18(2):59-66.

Walter A, Mayer C. Chapter 6: Peptidoglycan structure, biosynthesis, and dynamics during bacterial growth. In: Cohen E, Merzendorfer $\mathrm{H}$, editors. Extracellular Sugar-Based Biopolymers Matrices. Berlin: Springer; 2019. p. 237-99.

Weidel W, Pelzer H. Bagshaped Macromolecules - A new outlook on bacterial cell walls. Adv Enzymol Relat Subj Biochem. 1964;26:193232

Wyss C. Dependence of proliferation of Bacteroides forsythus on exogenous $\mathrm{N}$-acetylmuramic acid. Infect Immun. 1989 Jun;57(6):1757-9. 